\title{
Prediction of BeiDou Satellite Orbit Maneuvers to Improve the Reliability of Real-Time Navigation Products
}

\author{
Zhiwei Qin ${ }^{1} \mathbb{1}$, Le Wang ${ }^{1, *}$, Guanwen Huang ${ }^{1}$, Qin Zhang ${ }^{1}$, Xingyuan Yan ${ }^{2}$, Shichao Xie ${ }^{1}$, Haonan She ${ }^{1}$, \\ Fan Yue ${ }^{1}$ and Xiaolei Wang ${ }^{3}$ \\ 1 School of Geology Engineering and Geomatics, Chang'an University, 126 Yanta Road, Xi'an 710054, China; \\ gnssorbitqzw@chd.edu.cn (Z.Q.); huang830928@chd.edu.cn (G.H.); dczhangq@chd.edu.cn (Q.Z.); \\ wonderwall@chd.edu.cn (S.X.); lamme@chd.edu.cn (H.S.); south_wind@chd.edu.cn (F.Y.) \\ 2 School of Geospatial Engineering and Science, Sun Yat-sen University, No. 135, Xingang Xi Road, \\ Guangzhou 510275, China; yanxy35@mail.sysu.edu.cn \\ 3 School of Earth Sciences and Engineering, Hohai University, 1 Xikang Road, Nanjing 210098, China; \\ gnsswxl@hhu.edu.cn \\ * Correspondence: WangLe18@chd.edu.cn; Tel.: +86-029-8233-9043
}

check for updates

Citation: Qin, Z.; Wang, L.; Huang, G.; Zhang, Q.; Yan, X.; Xie, S.; She, H.; Yue, F.; Wang, X. Prediction of BeiDou Satellite Orbit Maneuvers to Improve the Reliability of Real-Time Navigation Products. Remote Sens. 2021, 13, 629. https://doi.org/ $10.3390 /$ rs13040629

Academic Editor: Ali Khenchaf Received: 20 January 2021

Accepted: 5 February 2021

Published: 9 February 202

Publisher's Note: MDPI stays neutral with regard to jurisdictional claims in published maps and institutional affiliations.

Copyright: (c) 2021 by the authors. Licensee MDPI, Basel, Switzerland. This article is an open access article distributed under the terms and conditions of the Creative Commons Attribution (CC BY) license (https:// creativecommons.org/licenses/by/ $4.0 /)$

\begin{abstract}
The positioning, navigation, and timing (PNT) service of the Global Navigation Satellite System (GNSS) is developing in the direction of real time and high precision. However, there are some problems that restrict the development of real-time and high-precision PNT technology. Satellite orbit maneuvering is one of the factors that reduce the reliability of real-time navigation products, especially the high-frequency orbit maneuvering of geostationary earth orbit (GEO) and inclined geosynchronous orbit (IGSO) satellites. The BeiDou Navigation Satellite System (BDS) constellation is designed to contain GEO, IGSO, and medium earth orbit (MEO). These orbit maneuvers bring certain difficulties for data processing, especially for BeiDou satellites, such as decreased real-time service performance, which results in real-time navigation products including unusable maneuvered satellites. Additionally, the performance of real-time navigation products will decrease because the orbit maneuvers could not be known in advance, which diminishes the real-time PNT service performance of BDS for users. Common users cannot obtain maneuvering times and strategies owing to confidentiality, which can lead to a decline in the BDS real-time service performance. Thus, we propose a method to predict orbit maneuvers. BDS data from the broadcast ephemeris were analyzed to verify the availability of the proposed method. In addition, the results of real-time positioning were analyzed by using ultra-rapid orbit products, demonstrating that their reliability is improved by removing maneuvered satellites in advance. This is vital to improve the reliability of real-time navigation products and BDS service performance.
\end{abstract}

Keywords: BeiDou satellites; orbit maneuvers; prediction; real-time navigation products

\section{Introduction}

The positioning, navigation, and timing (PNT) technology of the Global Navigation Satellite System (GNSS) grew out of nothing through development. The PNT service of GNSS is developing in the direction of real time and high precision. However, there are some problems restricting the development of real-time and high-precision PNT technology that decrease the reliability of real-time navigation products. Satellite orbit maneuvering is one of the factors that reduce the reliability of real-time navigation products, especially the high-frequency orbit maneuvers of geostationary earth orbit (GEO) and inclined geosynchronous orbit (IGSO) satellites. It is necessary to improve the reliability of the PNT service of GNSS in order to satisfy the demands of high-reliability and high-precision navigation products for users. Thus, it is vital to enhance the reliability of real-time navigation products (such as orbit, clock, etc.). Since 27 December 2012, BeiDou-2 has provided continuous PNT service for users in the entire Asia-Pacific region, with a constellation of five GEO, 
five IGSO, and four MEO satellites. The second phase, BeiDou-3, will provide PNT service for global users at the end of 2020 [1-3]. The successful launch of the last satellite is a milestone of the construction completed for the BeiDou-3 constellation. At present, there are 30 BeiDou-3 satellites shining in the sky, consisting of three GEO, three IGSO, and 24 MEO satellites. BDS is the only GNSS that contains GEO and IGSO satellites in the nominal satellite constellation, which is the highlight of this GNSS. The MEO satellites offer complete global coverage, sharing numerous common features with GPS and GLONASS. Despite the higher orbit of the GEO and IGSO satellites as compared with the legacy MEO constellations, BDS achieves fully adequate signal strength and competitive code and carrier tracking performance $[4,5]$. Together with GEO, IGSO and MEO satellites can provide fully global PNT service for worldwide users [6-10]. The GEO and IGSO satellites are considered relevant and valuable complements to GNSS services in the Asia-Pacific region, playing an important role [11-14]. They not only enhance satellite visibility and PNT availability for users in China and surrounding areas, but also improve PNT precision for global users. The estimation accuracy of the earth orientation parameters can be improved based on the different orbital altitudes of the BDS satellites [6]. However, due to gravitational perturbations from the Earth, Sun, and Moon, as well as other various perturbations to the satellites, orbit maneuvers are required at regular intervals to maintain predesignated positions. GEO and IGSO satellites are maneuvered more frequently than MEO satellites because they are geosynchronous and geostationary. When orbit maneuvering, the orbital elements are changed, which causes the status variable to change after maneuvering. The broadcast ephemeris includes the health status of GNSS satellites; their health identifier will be marked as 0 for healthy, and otherwise the satellite is unhealthy. The satellite health identifier from broadcast ephemeris is also marked as unhealthy for nonmaneuvered anomalies. Where information on the maneuvered satellites are unavailable owing to confidentiality, it brings several problems for GNSS data processing. With the development of Multi-GNSS, the position users can remove the unhealthy satellites directly, and the position accuracy could not be decreased. However, the orbit maneuvers and unmaneuvered abnormalities of satellites cannot be distinguished by using the SV health from broadcast ephemeris. They both would be marked as unhealthy. In addition, the unmaneuvered could not affect the precise orbit determination. However, the precise orbit of maneuvered satellites cannot be determined and predicted because of the extra maneuvered thrust. As the orbit maneuvers and unmaneuvered abnormalities cannot be distinguished, the ultra-rapid precise orbit products published by IGS analysis centers include the unusable maneuvered satellites. These problems decrease the reliability of high-precision real-time navigation products of BDS, such as the ultra-rapid precise orbit products published by the analysis center of the international GNSS Monitoring and Assessment System (iGMAS) and the Multi-GNSS Experiment (MGEX). The time information of maneuvered satellites is unavailable in advance, so the real-time navigation products contain the unusable orbits of maneuvered satellites. Unusable real-time navigation products that include maneuvered satellites will decrease the real-time PNT performance of BDS. In order to improve the reliability of the real-time navigation products, the time information of orbit maneuvers must be obtained in advance. The predicted information for orbit maneuvering can be provided for position strategy changes and precise orbit determination. Thus, it is vital to provide orbit maneuver information in order to remove unusable maneuvered satellites, which is crucial for real-time navigation product solutions and real-time positions.

Previous studies have investigated orbit maneuvers, yielding certain useful results. For orbit maneuvering of an inertial device to rendezvous and dock with another spacecraft, Lemmens et al. [15] and $\mathrm{Li}$ et al. [16] presented a maneuver detection method for LEO satellites based on historical two-line element (TLE) data. LEO satellite maneuvers can be relatively easily handled with precise Global Positioning System (GPS) or TLE data. Compared to LEO satellites, the maneuvering of navigation satellites is significantly more difficult to investigate due to their high altitudes and the missing positions of maneuvered satellites. These methods cannot be used to analyze GNSS orbit maneuvers. 
Huang et al. [17] and Cao et al. [18] used observations from the Chinese Area Positioning System (CAPS) to estimate the orbits of maneuvered GEO satellites. However, IGSO and MEO satellites cannot be continuously monitored via CAPS, so common users cannot obtain relevant observations. These methods bring certain difficulties when analyzing the characteristics of maneuvered orbits due to data limitations, especially for IGSO and MEO satellites. Huang et al. detected the start time of orbital maneuvers by using single point positioning (SPP) technology and residual values of a pseudo-range [19,20]. Qin et al. detected the orbit maneuvering time period by backward prediction of orbit and pseudo-range and determined the orbit for maneuvered satellites [21,22]. Numerous studies have investigated orbit maneuver detection and determination; however, there is a lack of studies specifically focusing on predicting orbit maneuvers. This is why we propose a prediction method for orbit maneuvers. In this study, we analyze BeiDou satellite orbit maneuvers to characterize orbital elements, including the relationship with the orbital semimajor axis. First, we analyzed long-term variations in the orbital semimajor axis and proposed a prediction method for east-west maneuvering. The experiment results based on broadcast ephemeris were analyzed to demonstrate the validity of the prediction method for orbit maneuvers. In addition, we analyzed the results of real-time positioning based on ultra-rapid precise orbit products from the German Research Centre for Geosciences (GFZ), and we present our conclusions and implications.

\section{Orbital Maneuver Analysis and Prediction Method}

Orbital elements have changed continuously due to perturbations from multiple planets and spacecraft, which cause long-term satellite drift. Satellites tend to gradually deviate from their designed orbits due to various perturbations, including perturbations from solar radiation pressure, Earth's nonspherical gravity, and the gravity of the Sun, Moon, Venus, Mars, and other planets. External forces are solar, and other forces come from places such as nonspherical bodies. The total forces are calculated by:

$$
F_{t}=\sum F_{g_{i}}+F_{S}+\sum F_{n_{i}}
$$

where $F_{t}$ is total forces affecting satellites, $F_{g_{i}}$ is gravity from other planets, $F_{S}$ is the force from solar radiation pressure, and $F_{n_{i}}$ is the force of nonspherical body [23]. There are two modes of orbit maneuvers: east-west (in plane) maneuvers can retain the shape of the orbit plane, and north-south (out of plane) maneuvers can retain the inclination of the orbit plane. Orbit maneuvers in the north-south direction are much less frequent than maneuvers in the east-west direction. Thus, orbit maneuvers in the north-south direction are not discussed in this study. A schematic diagram of the gravitational force from the Sun and other planets affecting satellites is shown in Figure 1.

In Figure 1, the orange circle and triangle denote the Sun, the yellow circle denotes Venus, and the dotted yellow line is its orbit around the Sun. The red circle denotes Mars, and its orbit is illustrated by a dotted red line. Earth is denoted by a green circle, and the dotted green line denotes its orbit around the Sun. The blue line denotes the designed orbit for satellites around the Earth, and the blue object is a satellite. Other planets (Mercury, Jupiter, Saturn, Uranus, Neptune, and Moon) are not shown in Figure 1. The effect of instantaneous gravity from planets on satellites is denoted by arrows. The dotted orange arrow denotes the joint forces of gravity and solar pressure from the Sun. The dotted yellow, red, and green arrows denote the gravity from Venus, Mars, and Earth, respectively. Supposing the force affecting satellites is only from the Earth and the density of the Earth is uniform, satellites could run perennially around the Earth in their designed orbits; the blue arrow denotes the velocity of satellites. In fact, there are various forces acting on satellites from the gravity of multiple planets, solar pressure, and nonspherical gravity from the Earth. Thus, the actual instantaneous joint forces acting on satellites are different with the gravity from the Earth, which is denoted by the dotted black arrow, and the actual instantaneous velocity is expressed by the solid black arrow. Satellite orbits are 
continuously changed due to perturbations from multiple planets and other factors, as shown in Figure 2.

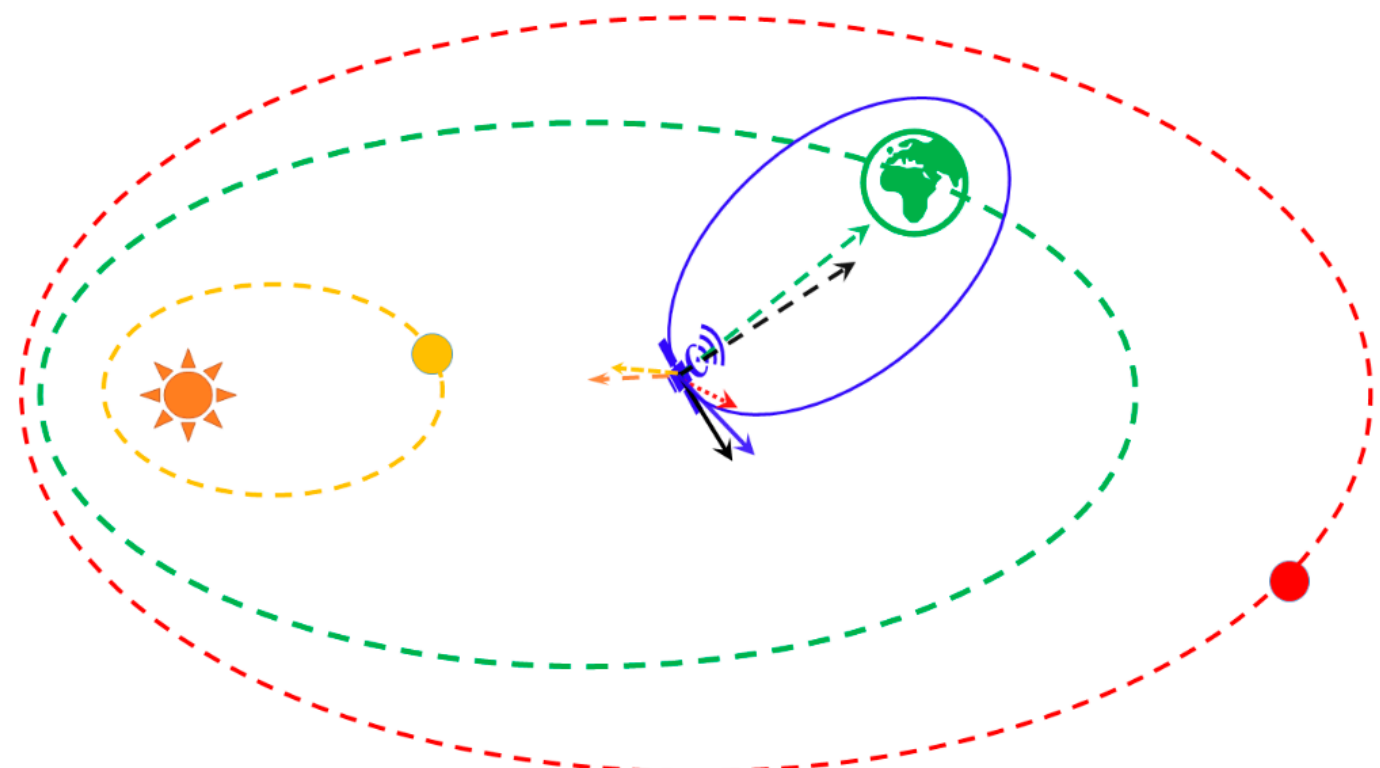

Figure 1. Schematic diagram of gravitational force from planets affecting satellites.

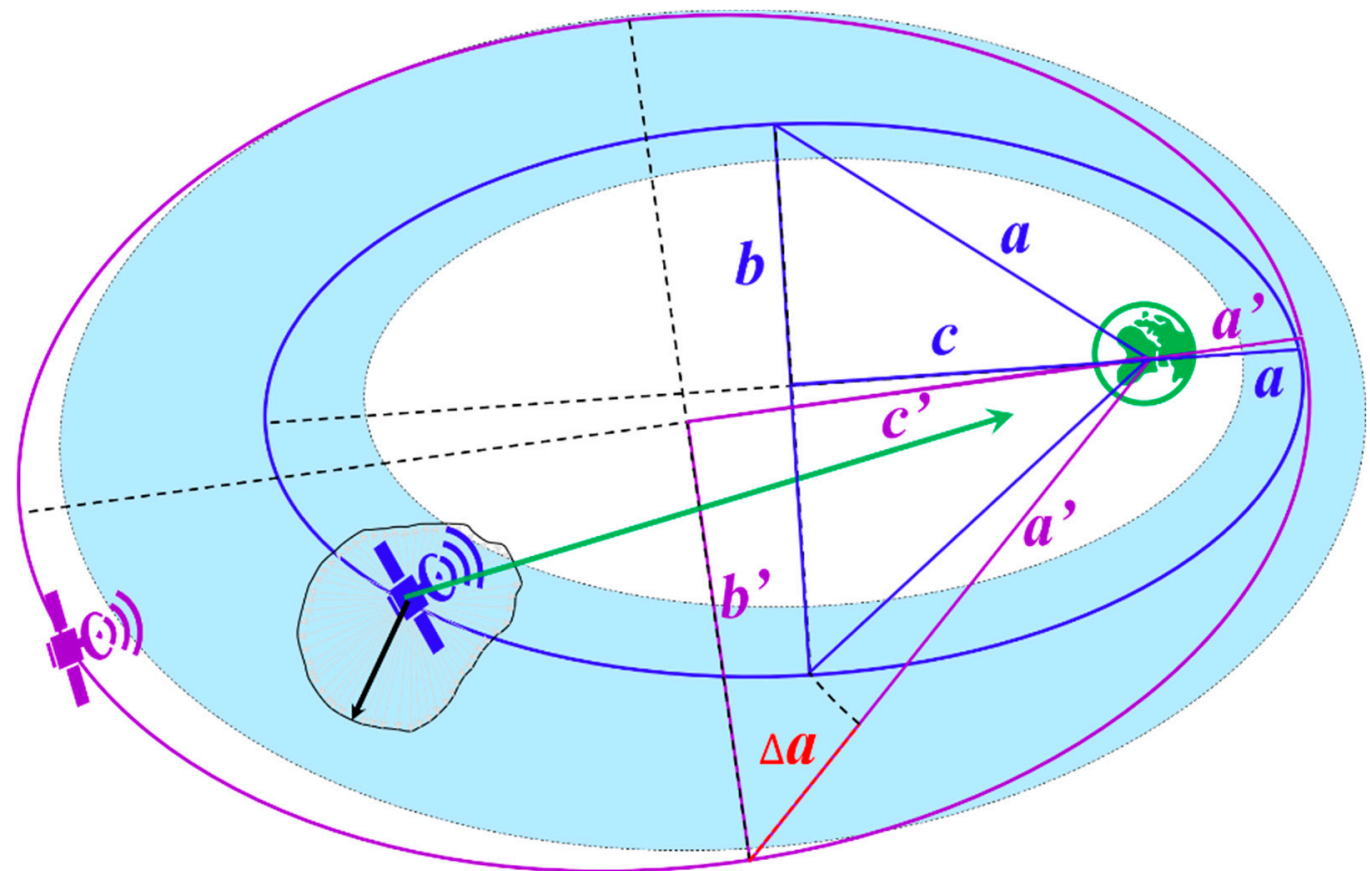

Figure 2. Schematic diagram of satellite orbit drift due to various perturbations from multiple planets and spacecraft.

In Figure 2, the green circle denotes the Earth, which is the focal point of satellite orbits. The initial orbit of satellites is shown the elliptical blue curve, line $a$ is the orbital semimajor axis, line $b$ is the orbital semiminor axis, and line $c$ is the half focal length of the satellite orbit. The light blue part denotes the normal range of satellite orbits. When satellites deviate from the normal orbit range, they can be adjusted by orbit maneuvering, using their propulsion system. The green arrow denotes the instantaneous gravity force acting on satellites from the Earth. The black arrow denotes the instantaneous joint forces from multiple planets acting on satellites, which leads to satellites tending to gradually deviate from their normal orbital range. The shape of the satellite orbits also changes with 
time. This can be shown by the pure curve, which denotes the satellite orbit that needs to be adjusted though orbit maneuvering; line $a^{\prime}$ is the orbital semimajor axis, line $b^{\prime}$ is the orbital semiminor axis, and line $c^{\prime}$ is the half focal length of the satellite orbit. The variation in the orbital semimajor axis $\Delta a$ is the difference value of the norm of $a$ and $a^{\prime}$, which is indicated by the dotted red line in Figure 2. Satellites periodically traverse the sky around the center of the Earth, which allows the perturbations from multiple planets to yield periodicity. The comprehensive perturbations from planets on satellites can be counteracted on short-term time scales [24]. The short-term periodical variation of the semimajor axis is not discussed for orbit maneuver prediction in this study. The comprehensive perturbations also cause long-term satellite drift, which leads to variation of the orbital semimajor axis. The orbital semimajor axis $a$ can be expressed as follows:

$$
a\left(x_{j}\right)=a_{0}+f\left(x_{j}\right)+\delta_{j},
$$

where $a_{0}$ is the initial value of the orbital semimajor axis; $f\left(x_{j}\right)$ is the long-term variation function for the orbital semimajor axis; $x_{j}$ is the time of the epoch, i.e., the day of the year containing the hours used; $\delta_{j}$ is the error associated with the orbital semimajor axis, which includes short-term periodical variation, random error, and calculation error from broadcast ephemeris; $a\left(x_{j}\right)$ is the value at epoch $x_{j}$, for which we used data from the broadcast ephemeris. According to the effects of joint forces from multiple planets acting on satellites, the long-term variation in the orbital semimajor axis can be described by a polynomial function as follows:

$$
f\left(x_{j}\right)=\sum_{p=1}^{n} k_{a} x_{j}^{p},
$$

where $n$ is the power of the polynomial function, which depends on the data fitting results, and $k_{a}$ is the multinomial coefficient. The remaining symbols have the same meaning as described above.

The long-term rate of change for the orbital semimajor axis can be calculated using nonmaneuvered data based on Equation (3). The minimum values of the orbital semimajor axis before orbit maneuvers can be obtained from long-time historical data from broadcast ephemeris, which is defined as the warning value, $a_{w}$. The warning time of maneuvers can be calculated as follows:

$$
t_{w}=t_{0}+\frac{a_{w}-a_{0}}{k_{a}}
$$

where $t_{0}$ is the time of the initial orbital semimajor axis value; $a_{w}$ is the warning value of the east-west orbit maneuvers, which can be obtained from the statistical results; $a_{0}$ is the initial value of the orbital semimajor axis; $k_{a}$ is the long-term rate of the orbital semimajor axis, which can be calculated by data fitting; $t_{w}$ is the warning time of the maneuvers. This prediction method can be applied to GEO, IGSO, and MEO satellites.

However, the start and end times are controlled by the satellite control center and common users cannot obtain orbit maneuvering strategies owing to confidentiality. Due to short-term perturbations to satellites from multiple planets, the warning values for the orbital semimajor axis are inaccurate. Thus, the predicted warning time is the reference time required to change the strategy of real-time positioning and orbit determination for users. Before orbit maneuvering, the maneuvered satellites would be marked as unhealthy in broadcast ephemeris, and nonmaneuvered satellites may also be marked as unhealthy. Thus, the proposed prediction method is combined with the SV health from the broadcast ephemeris to improve the prediction accuracy. The orbit maneuver prediction factor of orbital semimajor axis is defined as $P_{a}$ :

$$
P_{a}=\left\{\begin{array}{l}
1 \\
0
\end{array}, \quad a_{T F}=\left\{\begin{array}{cl}
\text { True } & a_{i}>a_{w} \\
\text { False } & a_{i} \leq a_{w}
\end{array},\right.\right.
$$


where $P_{a}$ is the prediction factor of the orbital semimajor axis at epoch $i ; a_{i}$ is the orbital semimajor axis value of epoch $i ; a_{w}$ is the warning value of the orbital semimajor axis; $a_{T F}$ is the warning flag of the orbital semimajor axis, which is true when the value of $a_{i}$ is greater than $a_{w}$, otherwise $a_{T F}$ is false. The orbit maneuver prediction factor of the satellite health identifier is defined as follows:

$$
P_{H}=\left\{\begin{array}{l}
1 \\
0
\end{array}, H_{T F}=\left\{\begin{array}{cc}
\text { True } & H_{S} \neq 0 \\
\text { False }
\end{array}, H_{S}=0,\right.\right.
$$

where $P_{H}$ is the prediction factor of the health identifier from broadcast ephemeris at epoch $i$, and $H_{S}$ is the health identifier from the broadcast ephemeris; 0 indicates that the satellite is healthy and the warning flag $H_{T F}$ is false, otherwise the satellite is unhealthy and $H_{T F}$ is true. The orbit maneuver prediction factor of the satellite health identifier is defined as follows:

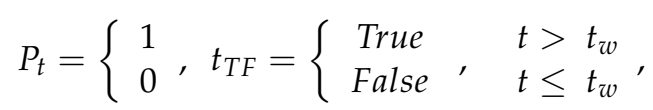

where $P_{t}$ is the prediction factor of the warning time at epoch $i ; t$ is the epoch time and $t_{w}$ is the warning time predicted for the orbit maneuvers; $t_{T F}$ is the warning flag of the epoch time for orbit maneuvering, which is true when $t$ is greater than $t_{w}$, otherwise $t_{T F}$ is false. The orbit maneuver prediction factor is defined as follows:

$$
P_{i}=P_{a}+P_{H}+P_{t}
$$

where $P_{i}$ is the orbit maneuver prediction factor at epoch $i$, which is unrelated in every epoch; when $P_{i}=2$, the satellite may be adjusted through orbit maneuvering several days later. Additionally, when $P_{i}=3$, the satellite can be adjusted in 1-2 h later, at the same time, we suggest that the analysis centers should remove the satellite from real-time navigation products before being published. The prediction information of orbit maneuvers is significant for calculating real-time navigation products, which dramatically improves the products' reliability.

\section{Experiment Analysis and Results}

To verify the proposed methods for analyzing the variation characteristics of orbital semimajor axis and orbit maneuver prediction, experimental data from the broadcast ephemeris were collected for processing. Due to the length limitation of the paper, we analyzed the variation characteristics of the orbital semimajor axes for GEO satellites and IGSO satellites using C01 in Sections 3.1 and 3.2, respectively. Considering the normal orbit determination precision of IGSO satellites is higher than that of GEO satellites, the reliability of ultra-rapid products for IGSO was analyzed in this study, but the prediction method can be applied to all GEO and IGSO satellites. In the first part, the variation characteristics of orbital semimajor axis proposed in this study are confirmed through the measured data from broadcast ephemeris. Next, the validity of the proposed orbit maneuver prediction method is verified in second part. Finally, we note that the reliability of ultra-rapid precise orbit products could be dramatically improved by using the prediction results of orbit maneuvering. The results of real-time positioning also indicate that the service performance of ultra-rapid precise orbit products can be enhanced. In order to demonstrate the applicability of GEO and IGSO satellites, their results are shown in the first and second part, respectively.

\subsection{Validation for Variation Characteristics of Orbital Semimajor Axis}

For variation characteristics of the orbital semimajor axis, we analyzed the experimental results for BeiDou C01. The orbital semimajor axis from the broadcast ephemeris was selected to estimate the coefficients of the functions, and data from 2-27 January 2015 were selected for the fit; the results are shown in Figure 3. 


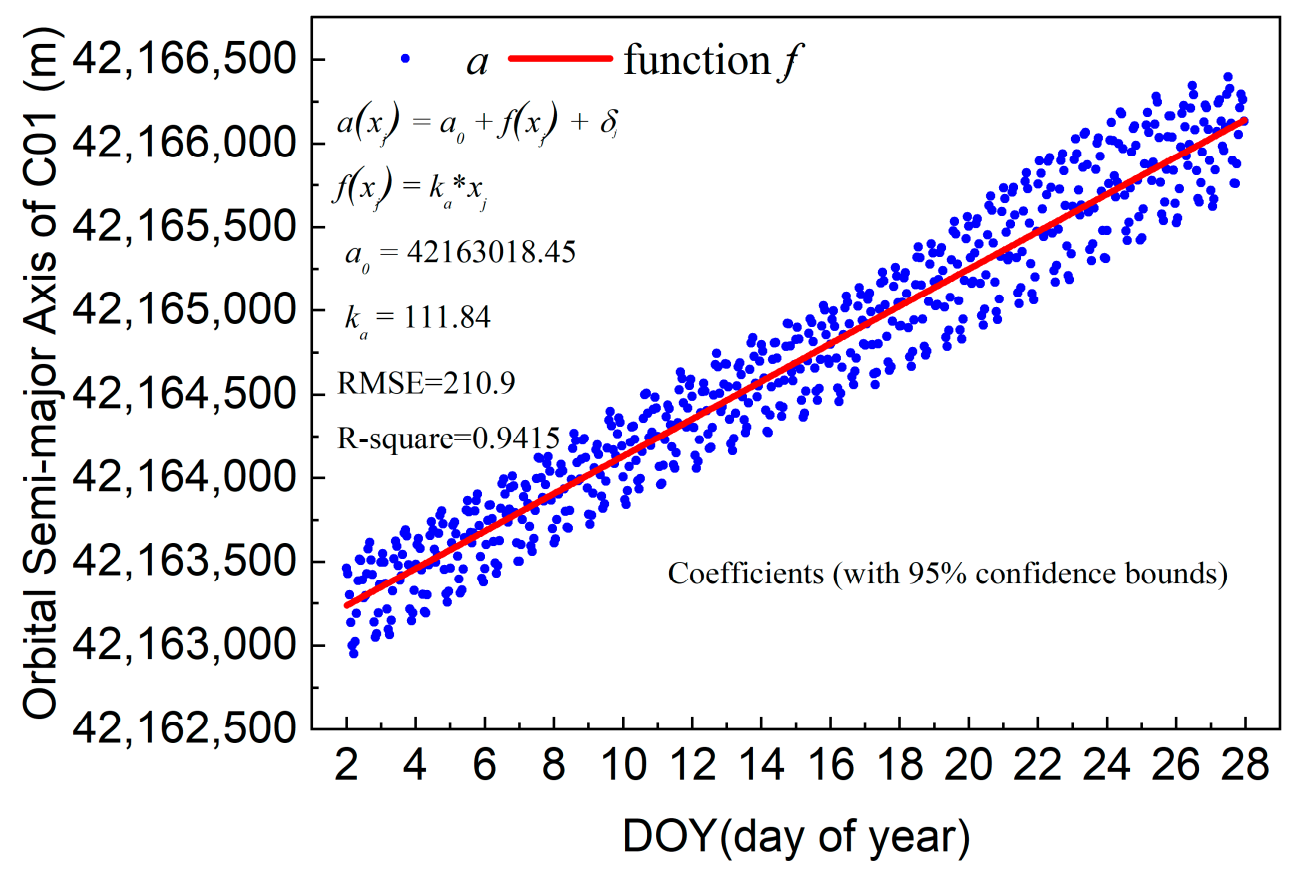

Figure 3. Time series values of orbital semimajor axis for C01 from 2-27 January 2015.

In Figure 3, the blue points indicate the time series values of the orbital semimajor axis for $\mathrm{C} 01$. The orbital semimajor axis trend is linear due to the long-term comprehensive influence from multiple planets. The red line indicates the function $f+a_{0}$, which is fitted using the data from 2-27 January 2015. The fitting coefficients of function $f$ are also shown in Figure 3: $a_{0}$ is $42,163,018.15 \mathrm{~m}$ and $k_{a}$ is $111.84 \mathrm{~m} /$ day. In addition, the measured orbital semimajor axis also includes short-term periodical variation of the orbital semimajor axis due to the short-term comprehensive influence from multiple planets, random error, and orbital semimajor axis calculation error, which is reflected in $\delta_{j}$ and root mean square error (RMSE) of $210.09 \mathrm{~m}$. The coefficient of determination (R-square) is 0.9415 , and the analyzed variation characteristics of the orbital semimajor axis is indicated by the data fitting results. In addition, time series values of the orbital semimajor axis for C01 from 11 February 2013 to 12 December 2019 were calculated.

In Figure 4, the blue points indicate the time series values of the orbital semimajor axis for C01. The orbital semimajor axis trend is linear due to the long-term comprehensive influence from multiple planets. The results demonstrate that the variation characteristics of orbital semimajor axis can be described as a linear function. From the results we can know that the slope may change after orbit maneuvers, which were consistent before maneuvers. Additionally, when the value of the orbital semimajor axis is greater than warning value, the long-term rate of the orbital semimajor axis and the warning time can be recalculated. 


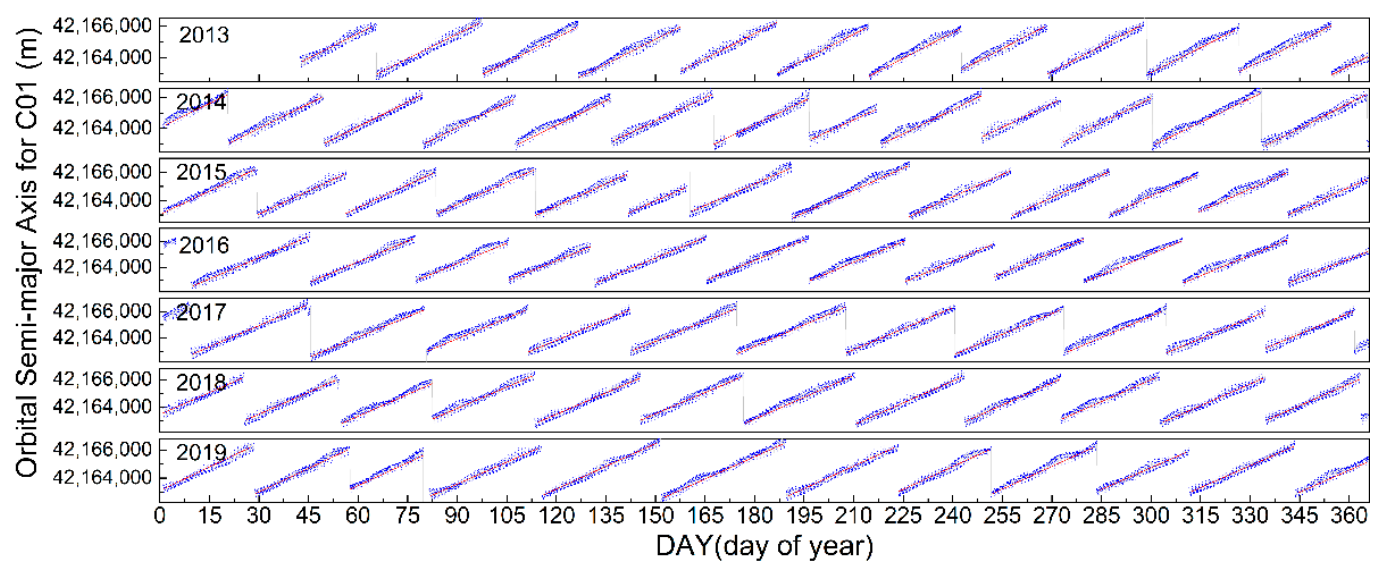

Figure 4. Time series values of orbital semimajor axis for C01 from 11 February 2013 to 12 December 2019.

\subsection{Validation of Orbit Maneuver Prediction Algorithm}

In this section, we demonstrate the availability of the maneuver prediction method proposed in this study. We analyzed the experimental results for BeiDou C07, and the orbital semimajor axis and SV health from broadcast ephemeris were selected for analysis. Broadcast ephemeris published by MGEX and iGMAS from 2013 to 2019 (7 y) was collected, which contains BeiDou data since 12 February 2013. We analyzed the C 07 satellite, and there were 13 instances of orbit maneuvers from 12 February 2013 to 10 March 2019. Before orbit maneuvering, the minimum value of the orbital semimajor axis was $42,171,060 \mathrm{~m}$, which is more appropriate as the warning value of orbit maneuvers $a_{w}$. The orbital semimajor axis was analyzed first, and the results are shown in Figure 4.

In Figure 5, the x-axis is the day of the year and the y-axis is the value of the orbital semimajor axis. The teal and black lines indicate the graph of the function. The function was fitted using data from 21 March to 13 July 2019; $a_{0}$ is $42,149,416.488 \mathrm{~m}$ and $k_{a}$ is $95.39 \mathrm{~m} /$ day. In addition, the measured orbital semimajor axis also includes short-term periodical variation due to the short-term comprehensive influence from multiple planets, random error, and orbital semimajor axis calculation error, which is reflected in $\delta_{j}$, and the root mean square error (RMSE) is $258.8 \mathrm{~m}$. The coefficient of determination (R-square) is 0.9934 . The value of $a_{w}$ is $42,171,060 \mathrm{~m}$ and the warning time of the maneuvers $t_{w}$ can be calculated as 15 August 2019 by Equation (4) The blue and purple points denote the measured orbital semimajor axis before and after orbit maneuvering, respectively. The C07 satellite was adjusted through orbit maneuvering on 23 August 2019. The orbit maneuvering mechanism is unknown, and the measured orbital semimajor axis contains the short-term comprehensive influence from multiple planets, random error, and orbital semimajor axis calculation error, which brings difficulties in accurately calculating the warning time of the maneuvers. Therefore, orbit maneuver prediction factors were analyzed, and the results are shown in Figure 6. 


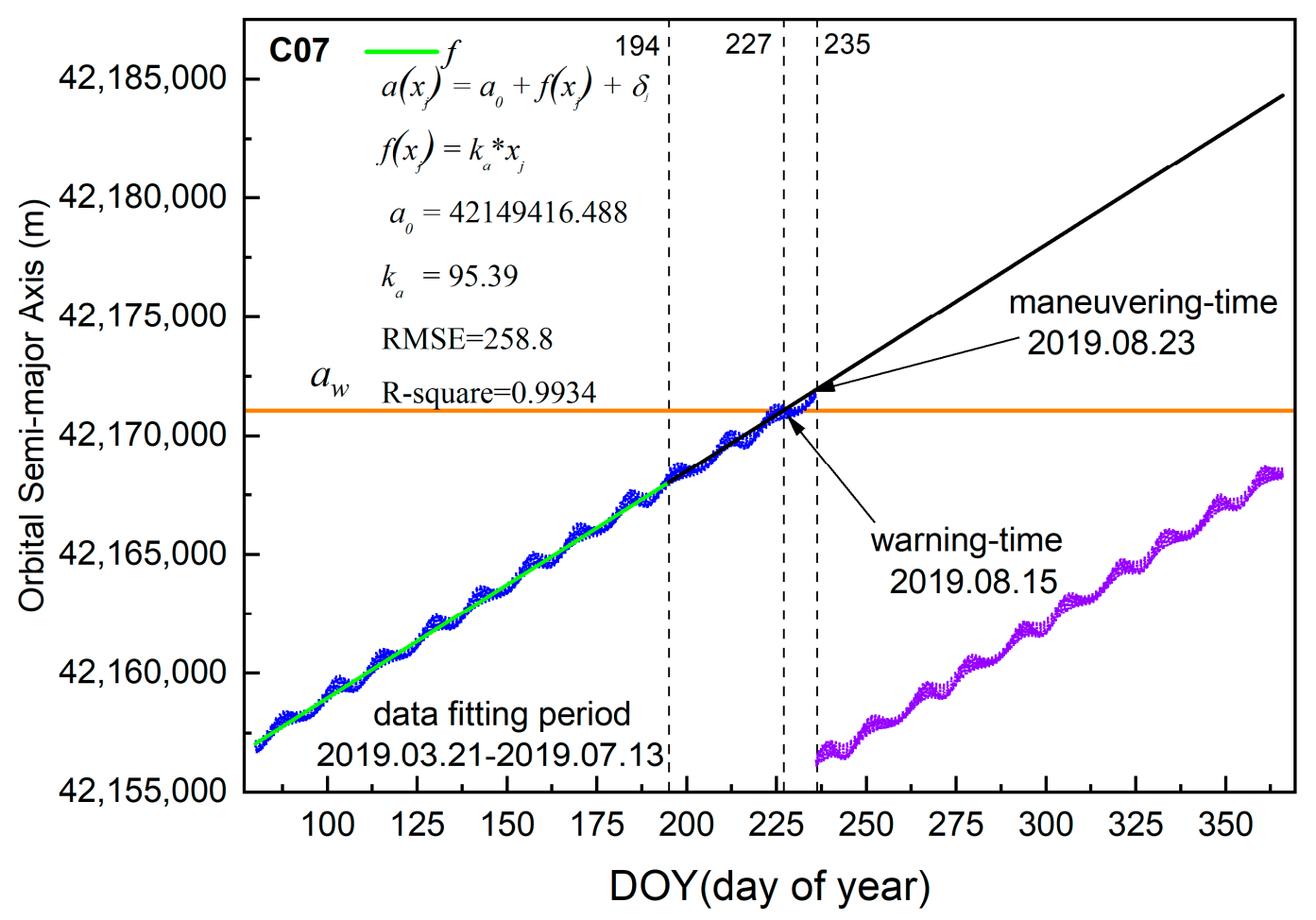

Figure 5. Analysis results of orbital semimajor axis for C07 satellite.

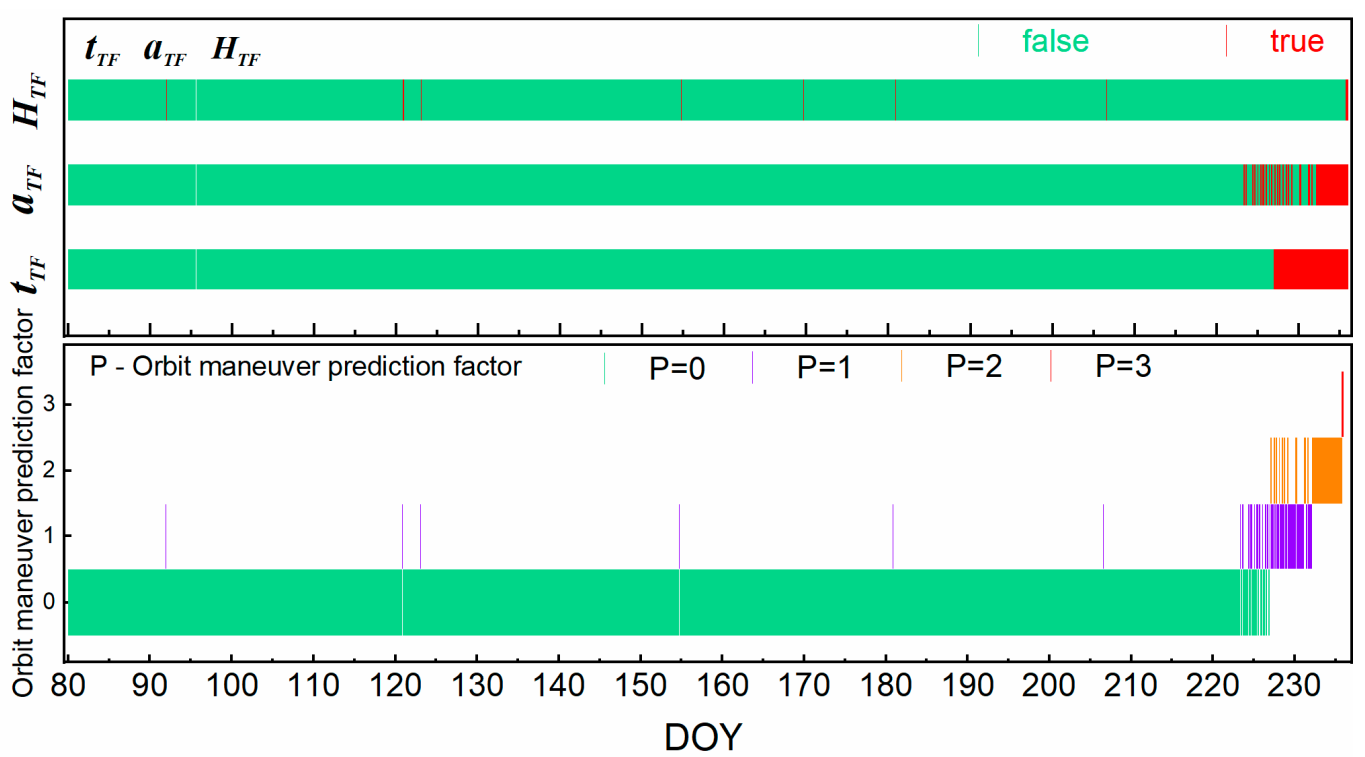

Figure 6. Orbit maneuver prediction factors for C07 satellite.

In Figure 6, the x-axis is the day of the year, and the top half is the results of warning flags of orbital semimajor axis and SV health and the warning time flag for orbit maneuvering. The green vertical bar indicates that the corresponding value is false, and red vertical bars indicate true values. The first line denotes the warning flag of SV health; C07 was sometimes marked unhealthy in this period. Satellites marked as unhealthy are not necessarily adjusted by orbit maneuvering, which needs the measured orbital semimajor axis and the warning time for orbit maneuvers to calculate the orbit maneuver prediction factors. The results of orbit maneuver prediction factors are shown in the bottom half, and a partial detail of Figure 6 is shown in Figure 7. 


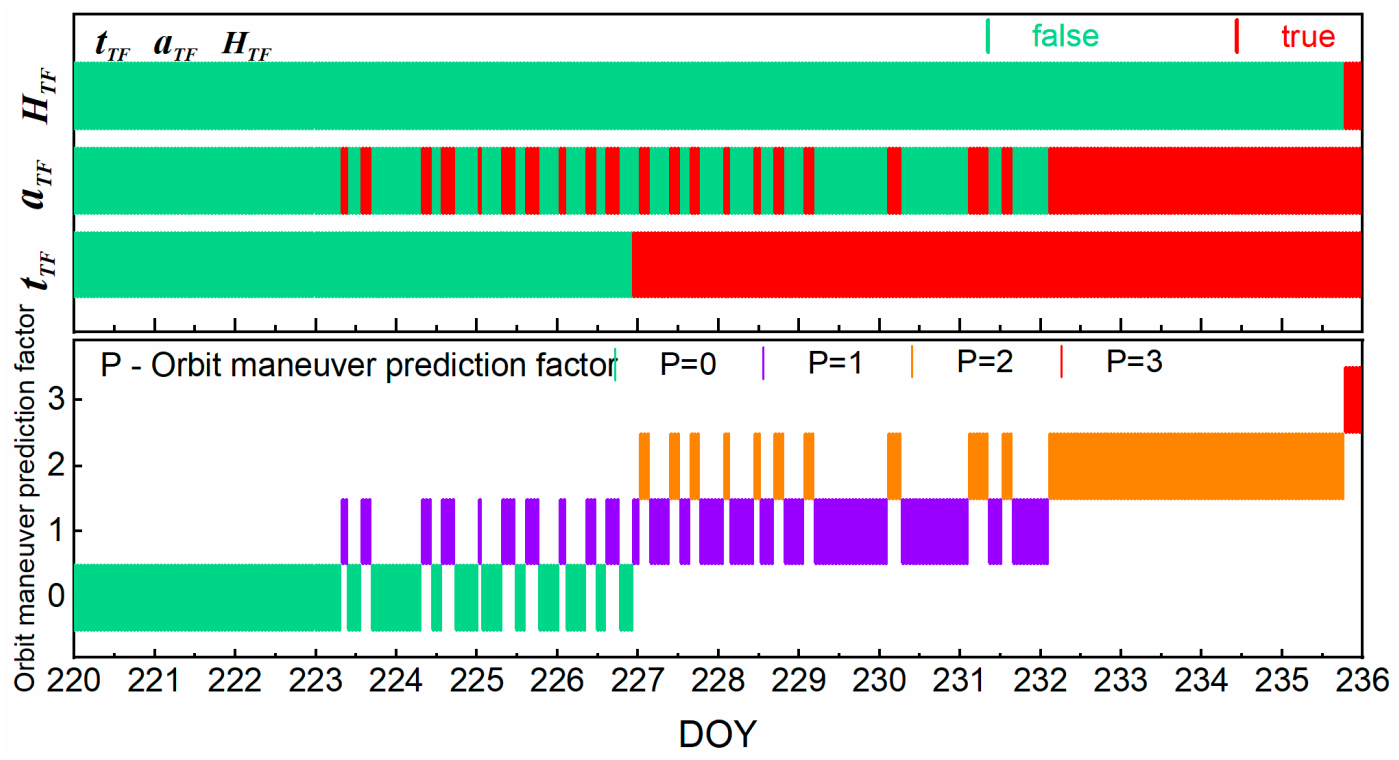

Figure 7. Orbit maneuver prediction factors for $\mathrm{C} 07$ satellite.

From Figure 7, it can be seen that the warning flag of the orbital semimajor axis appeared as true for the first time on 11 August 2019. Due to the short-term comprehensive influence from multiple planets and random error, the warning flag of the orbital semimajor axis was true or false intermittently until 20 August. The flag of warning time for orbit maneuvering changed to true on 15 August. The flag of SV health changed to true at 16:00:00 on 23 August.

The value of the orbit maneuver prediction factor $P$ is determined by the above flags, which is shown in the bottom part. The green vertical bars show that the value of the orbit maneuver prediction factor is 0 , indicating that the satellite is healthy. The purple vertical bars show that the value of the orbit maneuver prediction factor is 1 , indicating that there are some abnormalities of the satellites, and this case is defined as attention level. The alert level is defined when the value of the orbit maneuver prediction factor is 2 , which is denoted by vertical orange bars, indicating that the satellite was recently adjusted by orbit maneuvering. The vertical red bars show that the value of the orbit maneuver prediction factor is 3 and the alarm level for maneuvers, which indicates the satellite would be maneuvered 1-2 $\mathrm{h}$ later. The orbit maneuver prediction factor of C07 satellite changed to 3 at 16:00:00 on 23 August 2019. This indicates that the satellite would be maneuvered between 19:00:00 and 21:00:00 on 23 August. In order to demonstrate that the C07 satellite was maneuvered on 23 August 2019, the final precise orbit products from iGMAS were chosen. The final precise orbit cannot be estimated because of maneuvered thrust. The header of the precise orbit products published by iGMAS and Wuhan University (WHU) was shown in Figure 8.

\begin{tabular}{|c|c|}
\hline \# CP2019 & $\begin{array}{llllll}8 & 23 & 0 & 0 & 0.00000000\end{array}$ \\
\hline \#\# 0711 & 900.0000000058718 \\
\hline 91 & G01G02G03G05G06G07G08G09G10G11G12G13G14G15G16G17 \\
\hline+ & G19G20G21G22G23G24G25G26G27G28G2 \\
\hline+ & 07R08R09R11R12R13R14R15R16R. \\
\hline+ & $24 \mathrm{C} 01 \mathrm{C} 02 \mathrm{C} 03 \mathrm{C} 04 \mathrm{C} 05 \mathrm{C06C08} \mathrm{C} 09 \mathrm{C} 10 \mathrm{C} 11 \mathrm{C} 12 \mathrm{C} 13 \mathrm{C} 14 \mathrm{C} 1$ \\
\hline+ & E09E11E12E13E14E15E18E19E21E2 \\
\hline & $\begin{array}{llll}0 & 0 & 0 & 0\end{array}$ \\
\hline
\end{tabular}

Figure 8. The header of the precise orbit products published by iGMAS and WHU on 23 August 2019.

The header of the precise orbit products from iGMAS removed the C07 satellite, which is the secondary proof of the orbital maneuver for C07. The results can be demonstrated 
through the orbit maneuver detection methods proposed by Huang and Qin et al. [19-21]. In addition, in order to demonstrate the significance of orbit maneuver prediction for real-time navigation products and positioning, the real-time position results using ultrarapid precise products are presented in the next section. Considering the time system of products from iGMAS is BeiDou Time (BDT), the ultra-rapid precise products from GFZ are analyzed in this study.

\subsection{Validation of Reliability of Ultra-Rapid Products by Real-Time Position}

In order to demonstrate the performance of ultra-rapid products containing maneuvered satellites, the real-time position results were analyzed, and are shown in this section. The ultra-rapid precise orbit products published by MGEX and iGMAS include the unusable orbits of maneuvered satellites because the time information of orbit maneuvers cannot be acquired in advance. There are two schemes for real-time position. The differences between Scheme 1 and Scheme 2 were shown in Table 1.

Table 1. The differences between Scheme 1 and Scheme 2.

\begin{tabular}{cc}
\hline Items & Orbit and Clock Products \\
\hline Scheme 1 & Ultra-rapid precise products of 18:00 on 23 August 2019 published by GFZ \\
\hline Scheme 2 & $\begin{array}{r}\text { Ultra-rapid precise products of 18:00 on 23 August 2019 published by GFZ, } \\
\text { which removed the maneuvered C07 satellite }\end{array}$ \\
\hline
\end{tabular}

In Scheme 1, the real-time position used the ultra-rapid precise products of 18:00 on 23 August 2019 published by GFZ. The GFZ ultra-rapid precise products were also chosen for Scheme 2, which removed the maneuvered C07 satellite in advance according to the prediction information. The carried phase and code measurements of single BDS from station ULAB, which is located at Ulaanbaatar, Mongolia, were collected for positioning. The C07 satellite was adjusted through orbit maneuvering between 19:00:00 and 21:00:00 on 23 August, which had to be observed by station ULAB. The station distribution and the ground track of the $\mathrm{C} 07$ satellite are shown in Figure 9.

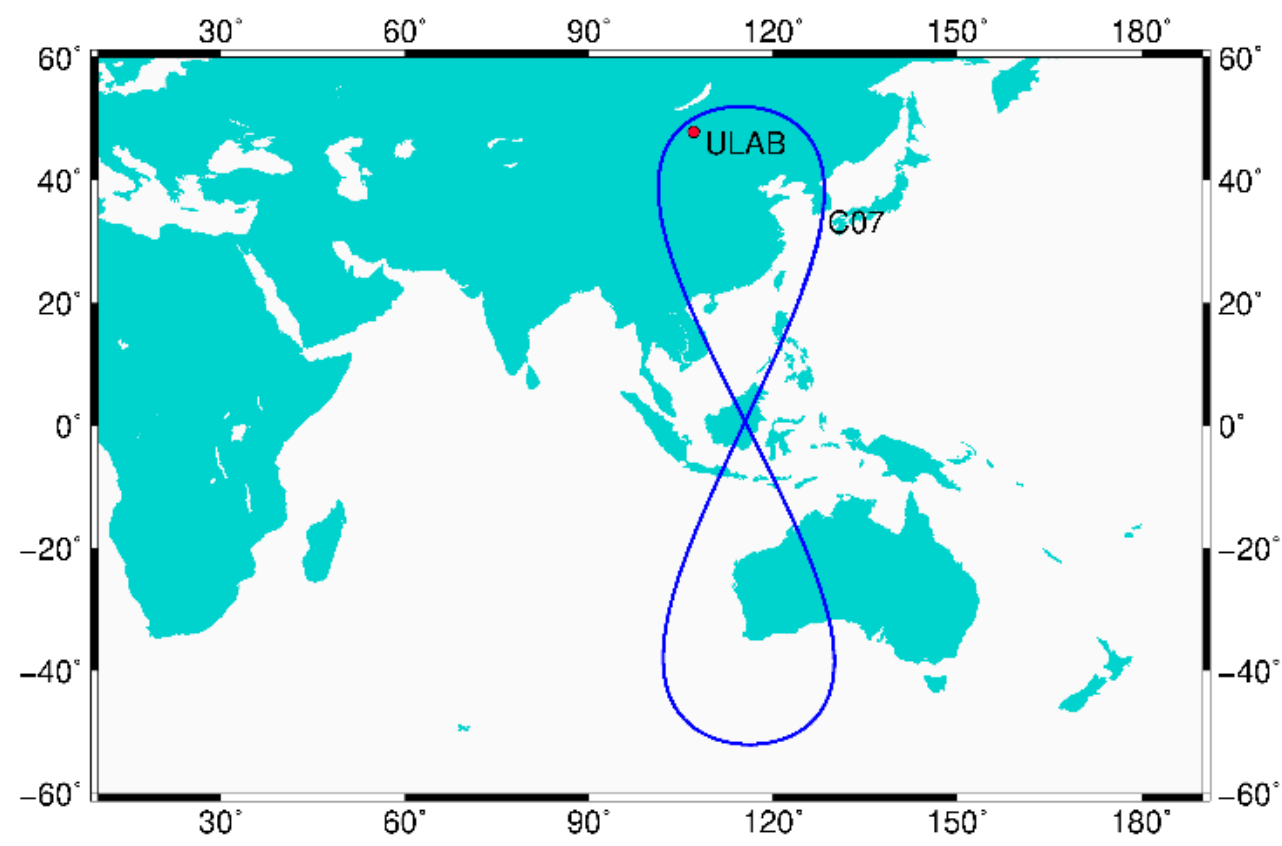

Figure 9. Station distribution and ground track of C07 satellite.

In Figure 9, the blue line denotes the ground track of the C07 satellite, which is distributed in the Asia-Pacific region as an 8 shape. The red point denotes the location of 
station $\mathrm{ULAB}$, which monitored the $\mathrm{C} 07$ satellite during the maneuvered period. There are two schemes for real-time positioning. The maneuvered C07 satellite contained in ultra-rapid orbit and clock products of GFZ for position in Scheme 1, which was removed in Scheme 2. The results of real-time precise point positioning (PPP) from 17:00:00-23:59:59 on 23 August 2019 are shown in Figure 10.

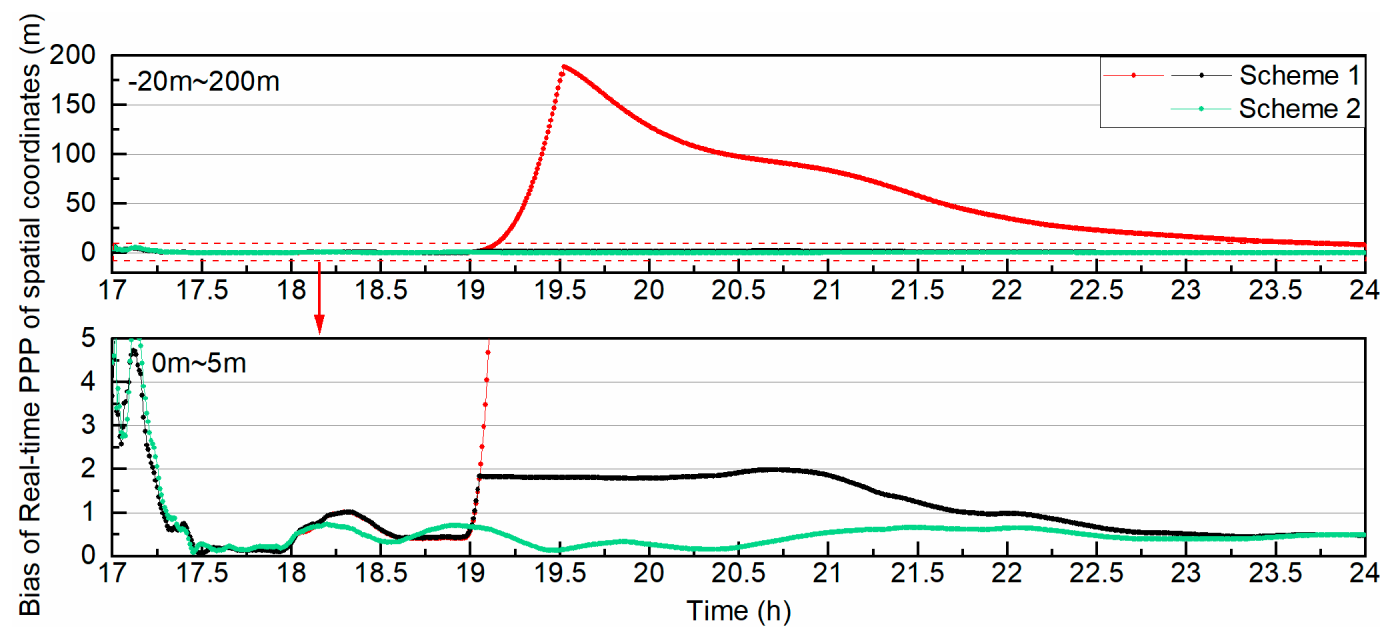

Figure 10. Bias of real-time precise point positioning (PPP) of spatial coordinates.

In Figure 10, the x-axis is the epoch time and the y-axis is the value of the bias of real-time PPP of spatial coordinates. The red points denote the real-time PPP bias after the first estimation of Scheme 1, which shows the error of the ultra-rapid precise products including the maneuvered satellite. The black points denote the real-time PPP bias after iterative estimation of Scheme 1, which indicates the real-time positioning accuracy of PPP by using the ultra-rapid precise orbit and clock products including the maneuvered satellite, and the green points denote the bias of Scheme 2. The bottom part is a detail of the top part. The biases of Schemes 1 and 2 are on the same order of magnitude before orbit maneuvering of the $\mathrm{C} 07$ satellite, which is less than $1.1 \mathrm{~m}$ after ambiguity resolution. The biases of both schemes changed differently after orbit maneuvering; the bias of Scheme 1 increased from 19:00:00 to 19:31:30, when the bias of the first estimation reached the highest point of $188.91 \mathrm{~m}$, then it decreased because the weight of $\mathrm{C} 07$ was reduced with the elevation angle. After iterative adjustments, the bias of Scheme 1 was less than $2 \mathrm{~m}$. The positioning biases in the east, north, and up directions are shown in Figures 11-13, respectively.

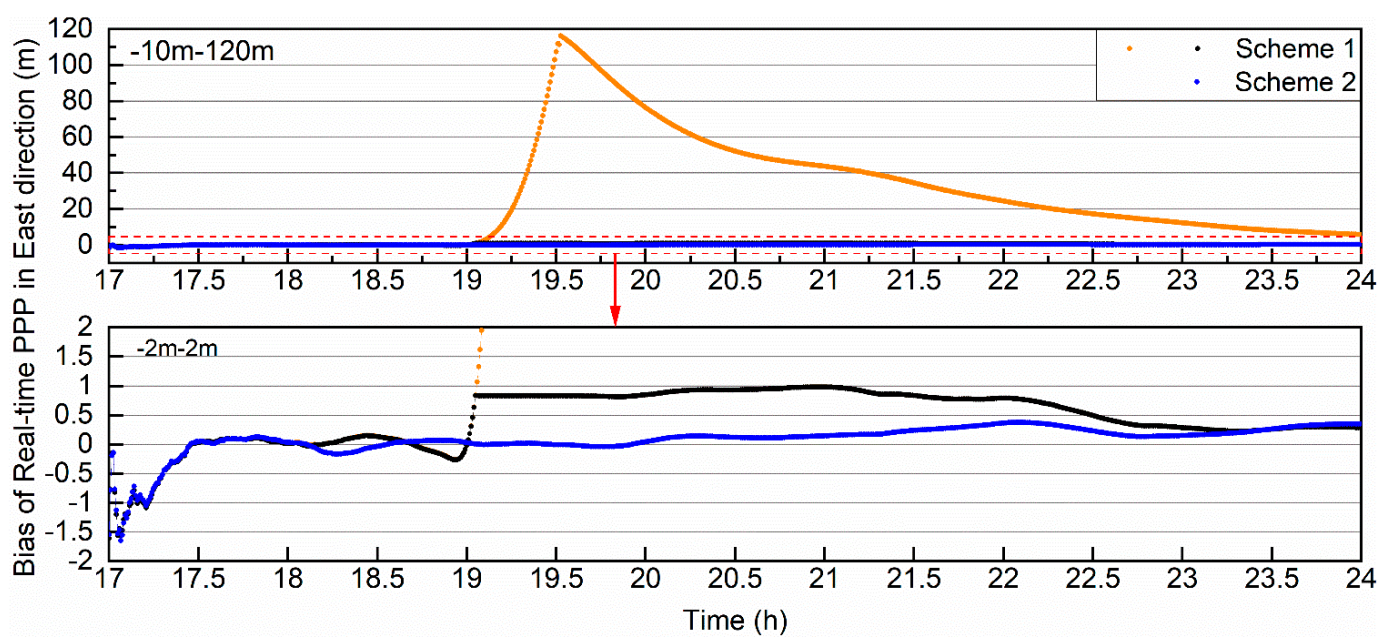

Figure 11. Bias of real-time PPP in the east. 


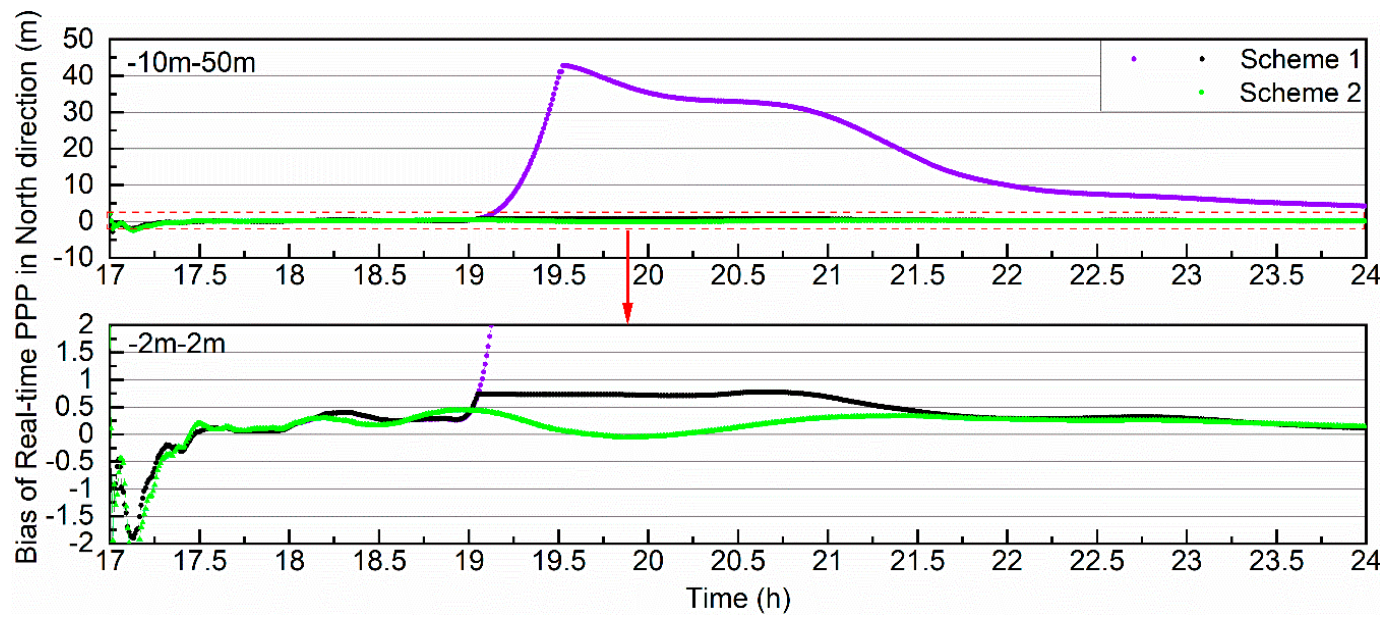

Figure 12. Bias of real-time PPP in the north.

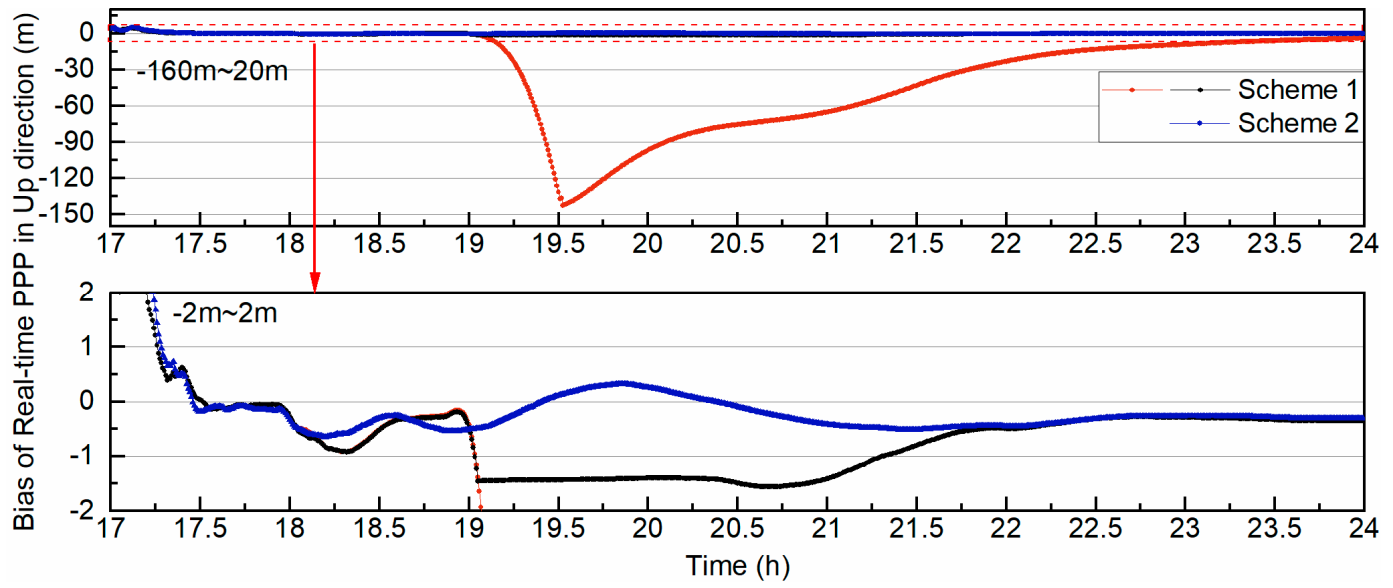

Figure 13. Bias of real-time PPP in the up direction.

In Figure 11, the x-axis is the epoch time and the y-axis is the value of the bias of real-time PPP in the east. The orange points denote the real-time PPP bias after the first estimation of Scheme 1, which shows the error of the ultra-rapid precise products including the maneuvered satellite. The black points denote the real-time PPP bias after iterative estimation of Scheme 1, which indicates the real-time positioning accuracy of PPP by using the ultra-rapid precise orbit and clock products including the maneuvered satellite, and the blue points denote the bias of Scheme 2. The bottom part is a detail of the top part. The biases of Schemes 1 and 2 are on the same order of magnitude before orbit maneuvering for the $\mathrm{C} 07$ satellite, which is less than $0.3 \mathrm{~m}$ after ambiguity resolution. The biases of both schemes changed differently after orbit maneuvering; the east bias of Scheme 1 increased from 19:00:00 to 19:31:30, when the bias of the first estimation reached the highest point of $116.26 \mathrm{~m}$, then it decreased because the weight of C07 was reduced with the elevation angle. After iterative adjustments, the bias of Scheme 1 was less than $1 \mathrm{~m}$. A comparison of the positioning biases between Schemes 1 and 2 is shown in Figure 14. 


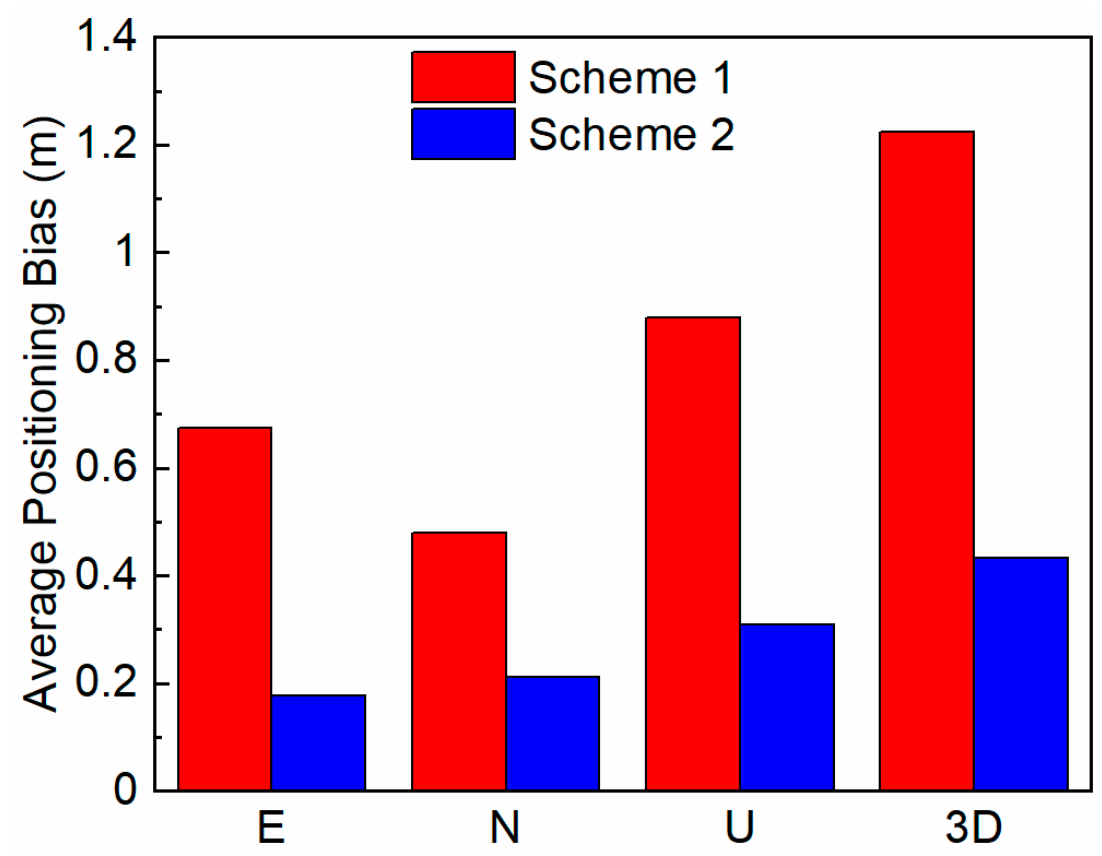

Figure 14. Average value of real-time PPP biases by using ultra-rapid precise products.

In Figure 12, the x-axis is the epoch time and the y-axis is the value of the bias of real-time PPP in the north. The purple points denote the real-time PPP bias after the first estimation of Scheme 1, the black points denote the real-time PPP bias after iterative estimation of Scheme 1, and the teal points denote the bias of Scheme 2. The bottom part is a detail of the top part. The biases of Schemes 1 and 2 are on the same order of magnitude before orbit maneuvering for the C07 satellite, which is less than $0.3 \mathrm{~m}$ after ambiguity resolution. The biases of both schemes changed differently after orbit maneuvering; the north-direction bias of Scheme 1 increased from 19:00:00 to 19:31:30, when the bias of the first estimation reached the highest point of $42.86 \mathrm{~m}$, then it decreased because the weight of $\mathrm{C} 07$ was reduced with the elevation angle. After iterative adjustments, the bias of Scheme 1 was less than $0.8 \mathrm{~m}$. A comparison of the positioning biases between Schemes 1 and 2 is shown in Figure 14.

In Figure 13, the x-axis is the epoch time and the y-axis is the value of the bias of real-time PPP in the up direction. The crimson points denote the real-time PPP bias after the first estimation of Scheme 1, the black points denote the real-time PPP bias after iterative estimation of Scheme 1, and the dark blue points denote the bias of Scheme 2. The bottom part is a detail of the top part. The biases of Schemes 1 and 2 are on the same order of magnitude before orbit maneuvering for the C07 satellite, which was less than $1 \mathrm{~m}$ after ambiguity resolution. The biases of both schemes changed differently after orbit maneuvering; the up bias of Scheme 1 increased from 19:00:00 to 19:31:30, when the bias of the first estimation reached the highest point of $142.60 \mathrm{~m}$, then it decreased because the weight of $\mathrm{C} 07$ was reduced with the elevation angle. After iterative adjustments, the bias of Scheme 1 was less than $1.6 \mathrm{~m}$. A comparison of the positioning biases between Schemes 1 and 2 is shown in Figure 14.

Figure 14 shows the average positioning biases in space and in the east, north, and up directions of station ULAB. The red columns denote the results of Scheme 1 and the blue columns are the results of Scheme 2 . In the east direction, the average bias of Scheme 1 is $0.67 \mathrm{~m}$ and of Scheme 2 is $0.18 \mathrm{~m}$, which was improved by $0.50 \mathrm{~m}(73.8 \%)$. In the north direction, the average bias of Scheme 1 is $0.48 \mathrm{~m}$ and of Scheme 2 is $0.21 \mathrm{~m}$, which was improved by $0.27 \mathrm{~m}(55.9 \%)$. In the up direction, the average bias of Scheme 1 is $0.88 \mathrm{~m}$ and of Scheme 2 is $0.31 \mathrm{~m}$, which was improved by $0.57 \mathrm{~m}(64.9 \%)$. In the three directions, the improvement in the north is more obvious when using the ultra-rapid products and removing the maneuvered $\mathrm{C} 07$ satellite, and the improvement in the east is imperceptible. 
It is related to the positional correlation of the orbit maneuvering direction and the station position. In the three dimensions, the average bias of Scheme 1 is $1.22 \mathrm{~m}$ and of Scheme 2 is $0.43 \mathrm{~m}$, which was improved by $2.17 \mathrm{~m}(64.7 \%)$.

From the above results, it is obvious that the reliability of ultra-rapid orbit products can obviously be improved without maneuvered satellites. The unusable orbits of maneuvered satellites in these products can bring some problems for data preprocessing and ambiguity resolution, which causes the phase measurements to be unusable. The real-time positioning results using the ionosphere-free code observations of station ULAB are shown in Figure 15.

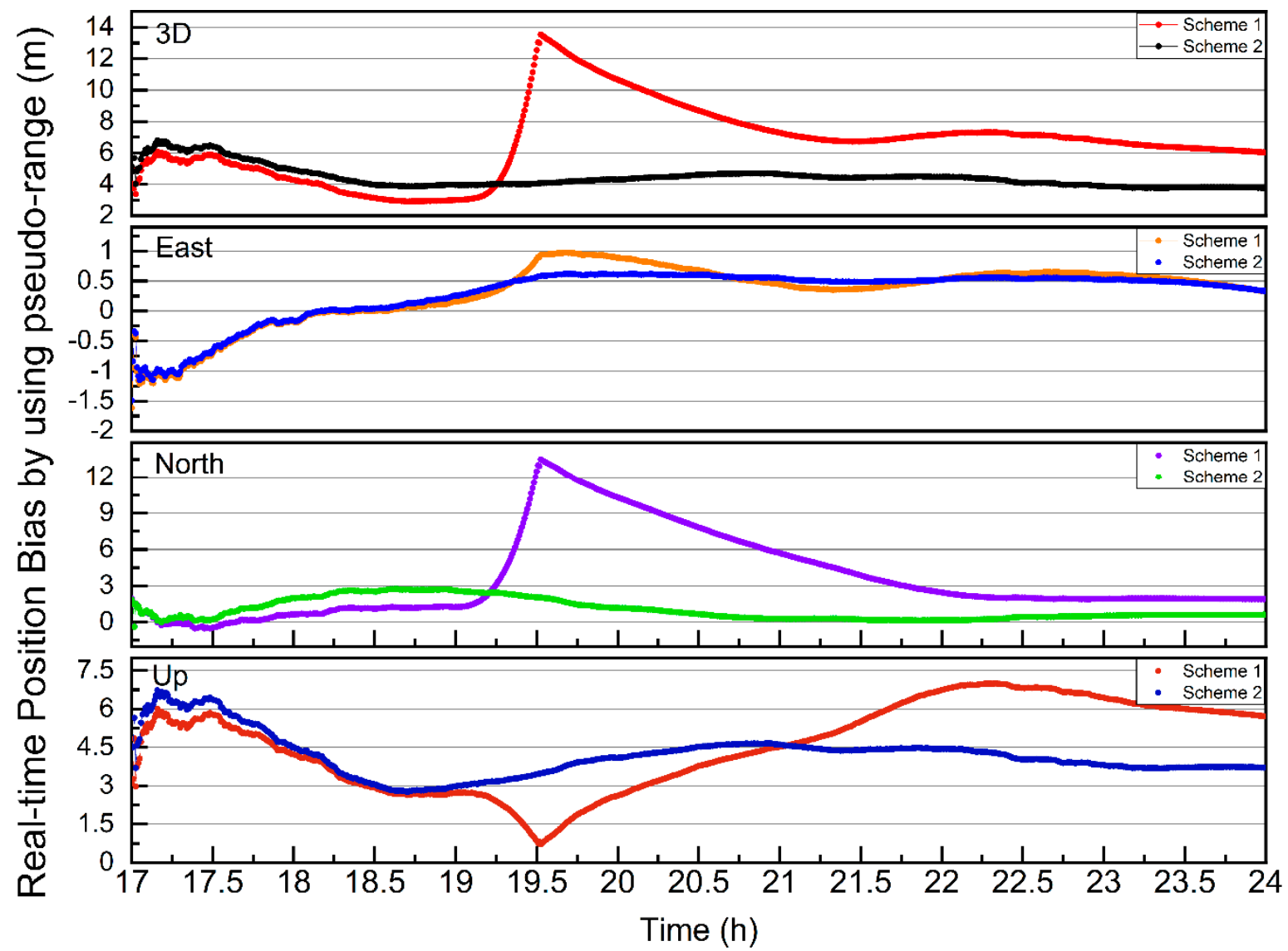

Figure 15. Results of real-time positioning by using ionosphere-free code observations.

In Figure 15, the x-axis is the epoch time and the $y$-axis is the value of real-time positioning bias by using the ionosphere-free code observations, which includes the results in space and in the east, north, and up directions. The red points denote the spatial positioning biases of Scheme 1 and the black points denote the results of Scheme 2 . The biases of both schemes decreased modestly before 7:00:00, and the bias of Scheme 1 is less than Scheme 2 due to more redundant observations. The biases of both schemes changed differently after orbit maneuvering; the spatial positioning bias of Scheme 1 increased from 19:00:00 to 19:31:30, when it reached the highest point of $13.54 \mathrm{~m}$, then it decreased because the weight of $\mathrm{C} 07$ was reduced with the elevation angle. The orange points denote the east positioning biases of Scheme 1 and the blue points denote the results of Scheme 2 . The results show that there were no more differences between the two schemes, because the positional correlation of the orbit maneuvering direction and the station position were randomized. The purple points denote the north direction positioning biases of Scheme 1 and the teal points denote the results of Scheme 2. The spatial positioning biases of Scheme 1 were less than Scheme 2 before 7:15:30 due to more redundant observations. The biases of both schemes changed differently after orbit maneuvering; the spatial positioning bias of Scheme 1 increased from 19:00:00 to 19:31:30, when it reached the highest point of 
$13.49 \mathrm{~m}$, then it decreased because the weight of C07 was reduced with the elevation angle. The crimson points denote the up direction biases of Scheme 1 and the dark blue points denote the results of Scheme 2 . The biases of both schemes changed differently after orbit maneuvering; the spatial positioning bias of Scheme 1 decreased before 19:31:30, when it reached the lowest point of $0.72 \mathrm{~m}$. As the positional correlation of the orbit maneuvering direction and the station position were randomized, the positioning bias in the up direction of Scheme 1 was less than Scheme 2 for station ULAB from 19:00:00 to 21:00:00 on 23 August 2019. The average value and standard deviation (STD) of positioning biases from 18:00:00 to 23:59:30 on 23 August 2019 are shown in Figure 16.

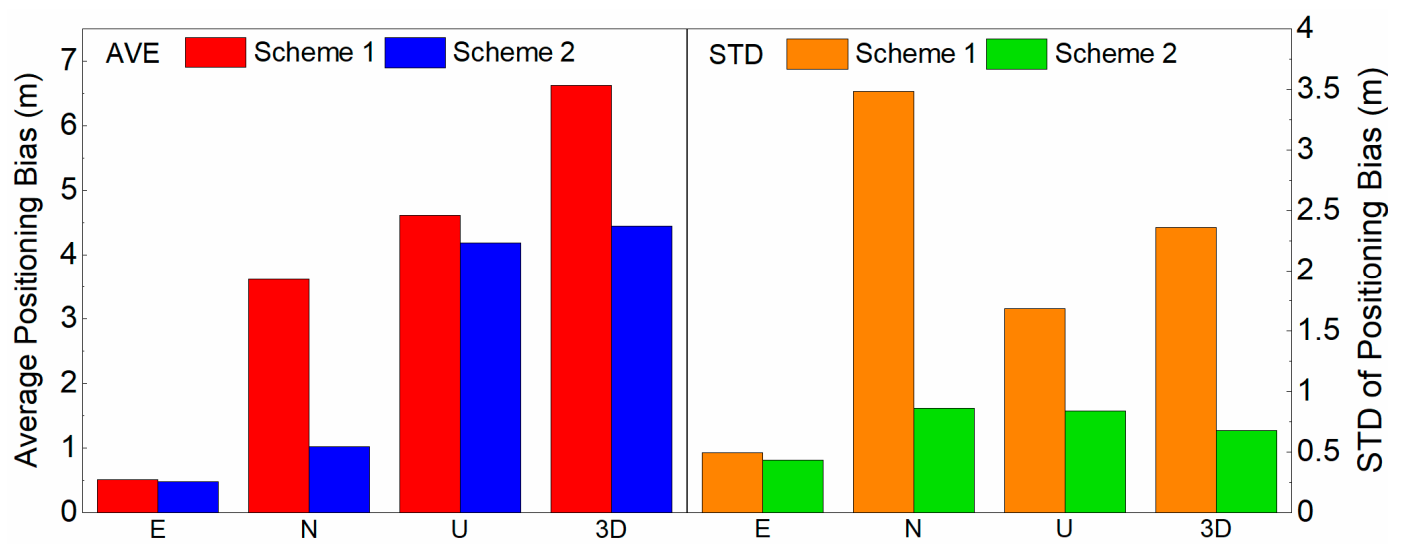

Figure 16. Average value and standard deviation (STD) of positioning biases by using ionosphere-free code observations.

In Figure 16, the graph on the left shows the average positioning bias of station ULAB. The red columns denote the results of Scheme 1 and the blue columns are the results of Scheme 2. In the east, the average bias of Scheme 1 is $0.51 \mathrm{~m}$ and of Scheme 2 is $0.47 \mathrm{~m}$, which was improved by $0.04 \mathrm{~m}(7.5 \%)$. The average bias of Scheme 1 is $3.61 \mathrm{~m}$ in the north and for Scheme 2 is $1.01 \mathrm{~m}$, which was improved by $2.60 \mathrm{~m}(71.9 \%)$. The average bias of Scheme 1 is $4.61 \mathrm{~m}$ in the up direction and of Scheme 2 is $4.19 \mathrm{~m}$, which was improved by $0.43 \mathrm{~m}(9.3 \%)$. In the three directions, the improvement in the north is more obvious when using the ultra-rapid products and removing the maneuvered C07 satellite, and the improvement in the east is imperceptible. This is related to the positional correlation of the orbit maneuvering direction and the station position. In the three dimensions, the average bias of Scheme 1 is $6.62 \mathrm{~m}$ and of Scheme 2 is $4.50 \mathrm{~m}$, which was improved by $2.17 \mathrm{~m}$ $(32.8 \%)$. The graph on the shows the standard deviation of positioning biases. The orange columns denote the results of Scheme 1 and the green columns are the results of Scheme 2 . The results of Scheme 1 are greater than Scheme 2 in all directions, which is obvious in the north. The spatial positioning biases of Scheme 1 are less than Scheme 2 before 7:15:30, but the standard deviation is greater.

The above results show that the real-time service performance of ultra-rapid precise products is improved by removing maneuvered satellites in advance. The orbit maneuver prediction method proposed in this study can provide alarm information of orbit maneuvers to remove maneuvered satellites from ultra-rapid precise orbit products. The reliability of ultra-rapid orbit products can be improved obviously without maneuvered satellites.

\section{Conclusions and Discussion}

In this study, we propose a prediction method for orbit maneuvers that can provide alarm information required to change the strategies for real-time navigation products and real-time positioning of maneuvered satellites. We analyzed the variation characteristics of the orbital semimajor axis of the satellites. Satellites tend to gradually deviate from their designed orbits due to various perturbations. The long-term variation in the orbital semimajor axis changes based on a linear trend, which can be described by a linear function. Based on the long-term variation characteristics of the orbital semimajor axis for satellites, a 
prediction method for orbit maneuvers is proposed, combined with SV health in broadcast ephemeris. BDS data from the broadcast ephemeris were analyzed to verify the availability of the proposed method. In addition, in order to demonstrate the significance of orbit maneuver prediction for real-time navigation products and positioning, we analyzed the results of real-time PPP and positioning using ionosphere-free code observations with ultra-rapid precise orbit products, demonstrating that the reliability of the products is improved by removing maneuvered satellites in advance. The orbit maneuver prediction method proposed in this study can provide alarm information of orbit maneuvers to remove maneuvered satellites from real-time navigation products. It is vital to improve the reliability of real-time navigation products and BDS service performance, and the method can also be applied to other GNSS.

However, there are some questions need to be discussed for removing the maneuvered satellites of real-time navigation products. The analysis centers of IGS and iGMAS can removed the maneuvered before publishing. However, the satellites may be maneuvered after products published. In this case, does the publishing strategy for ultra-rapid products need to be modified? At present, most of the analysis centers publish ultra-rapid products every three or six hours. Wuhan university publishes the ultra-rapid products once an hour, which can remove the maneuvered satellites timely to ensure the reliability of real-time navigation products. We suggest that the other analysis centers change original publishing strategy to provide the reliable real-time products for users.

Author Contributions: Z.Q., L.W., and G.H. provided the initial idea for this study; Z.Q., L.W., X.Y., and S.X. conceived and designed the experiments; G.H., Q.Z., Z.Q., and F.Y. analyzed the experimental results; Z.Q., G.H., H.S., and X.W. wrote the paper. All authors have read and agreed to the published version of the manuscript.

Funding: This work was funded by the Fundamental Research Funds for the Central Universities, CHD (300102260707), the Chinese Scholarship Council Studentship (ref. 202006560033), programs of the National Natural Science Foundation of China (No. 41774025, 41731066), the Special Fund for Technological Innovation Guidance of Shaanxi Province (2018XNCGG05), the Special Fund for Basic Scientific Research of Central Colleges (grant No. CHD300102268305, Chang'an University), the program of National Key Research and Development Plan of China (2018YFC1505102), and the Grand Projects of the Beidou-2 System (GFZX0301040308).

Institutional Review Board Statement: Not applicable.

Informed Consent Statement: Not applicable.

Data Availability Statement: The data can be downloaded from WHU (ftp://igs.gnsswhu.cn/) and Crustal Dynamics Data Information System (CDDIS, https:/ / cddis.nasa.gov/).

Acknowledgments: The authors would like to thank the MGEX, iGMAS, and IGS data center of Wuhan University for the data support.

Conflicts of Interest: The authors declare no conflict of interest.

\section{References}

1. Steigenberger, P.; Hugentobler, U.; Hauschild, A.; Montenbruck, O. Orbit and clock analysis of Compass GEO and IGSO satellites. J. Geod. 2013, 87, 515-525. [CrossRef]

2. Zhao, Q.; Guo, J.; Li, M.; Qu, L.; Hu, Z.; Shi, C.; Liu, J. Initial results of precise orbit and clock determination for COMPASS navigation satellite system. J. Geod. 2013, 87, 475-486. [CrossRef]

3. Li, X.; Ge, M.; Dai, X.; Ren, X.; Fritsche, M.; Wickert, J.; Schuh, H. Accuracy and reliability of multi-GNSS real-time precise positioning: GPS, GLONASS, BeiDou, and Galileo. J. Geod. 2015, 89, 607-635. [CrossRef]

4. Montenbruck, O.; Hauschild, A.; Steigenberger, P.; Hugentobler, U.; Teunissen, P.; Nakamura, S. Initial assessment of the COMPASS/BeiDou-2 regional navigation satellite system. GPS Solut. 2013, 17, 211-222. [CrossRef]

5. Lv, Y.; Geng, T.; Zhao, Q.; Liu, J. Characteristics of BeiDou-3 Experimental Satellite Clocks. Remote Sens. 2018, 10, 1847. [CrossRef]

6. Yang, Y.; Li, J.; Xu, J.; Tang, J.; Guo, H.; He, H. Contribution of the Compass satellite navigation system to global PNT users. Chin. Sci. Bull. 2011, 56, 2813-2819. [CrossRef]

7. Yang, Y.; Xu, Y.; Li, J.; Yang, C. Progress and performance evaluation of BeiDou global navigation satellite system: Data analysis based on BDS-3 demonstration system. Sci. China Earth Sci. 2018, 61, 614-662. [CrossRef] 
8. Huang, G.; Cui, B.; Zhang, Q.; Fu, W.; Li, P. An Improved Predicted Model for BDS Ultra-Rapid Satellite Clock Offsets. Remote Sens. 2018, 10, 60. [CrossRef]

9. Huang, G.; Cui, B.; Zhang, Q.; Li, P.; Xie, W. Switching and Performance Variations of On-Orbit BDS Satellite Clocks. Adv. Space Res. 2018, 63, 1681-1696. [CrossRef]

10. CSNO. BeiDou Navigation Satellite System Signal in Space Interface Control Document-Open Service Signal (Version 2.0.); China Satellite Navigation Office: Beijing, China, 2013.

11. Yang, Y. Progress, Contribution and Challenges of Compass/Beidou Satellite Navigation System. Acta Geod. Cartogr. Sin. 2010, 39, 1-6. [CrossRef]

12. He, L.; Ge, M.; Wang, J.; Wickert, J.; Schuh, H. Experimental Study on the Precise Orbit Determination of the BeiDou Navigation Satellite System. Sensors 2013, 13, 2911-2928. [CrossRef]

13. Huang, G.; Yan, X.; Zhang, Q.; Liu, C.; Wang, L.; Qin, Z. Estimation of antenna phase center offset for BDS IGSO and MEO satellites. GPS Solut. 2018, 22, 49. [CrossRef]

14. Zhang, X.; Wu, M.; Liu, W.; Li, X.; Yu, S.; Lu, C.; Wickert, J. Initial assessment of the COMPASS/BeiDou-3: New-generation navigation signals. J. Geod. 2017, 91, 1225-1240. [CrossRef]

15. Lemmens, S.; Krag, H. Two-Line-Elements-Based Maneuver Detection Methods for Satellites in Low Earth Orbit. J. Guid. Control Dyn. 2014, 37, 860-868. [CrossRef]

16. Li, T.; Li, K.; Chen, L. New manoeuvre detection method based on historical orbital data for low Earth orbit satellites. Adv. Space Res. 2018, 62, 554-567. [CrossRef]

17. Huang, Y.; Hu, X.; Huang, C.; Yang, Q.; Jiao, W. Precise orbit determination of a maneuvered GEO satellite using CAPS ranging data. Sci. China Ser. G Phys. Mech. Astron. 2009, 52, 346-352. [CrossRef]

18. Cao, F.; Yang, Y.; Li, Z.; Sun, B.; Kong, Y.; Chen, L.; Feng, C. Orbit determination and prediction of GEO satellite of BeiDou during repositioning maneuver. Adv. Space Res. 2014, 54, 1828-1837. [CrossRef]

19. Huang, G.; Qin, Z.; Zhang, Q.; Wang, L.; Yan, X.; Wang, X. A Real-Time Robust Method to Detect BeiDou GEO/IGSO Orbital Maneuvers. Sensors 2017, 17, 2761. [CrossRef] [PubMed]

20. Huang, G.; Qin, Z.; Zhang, Q.; Wang, L.; Yan, X.; Fan, L.; Wang, X. An Optimized Method to Detect BDS Satellites' Orbit Maneuvering and Anomalies in Real-Time. Sensors 2018, 18, 726. [CrossRef]

21. Qin, Z.; Huang, G.; Zhang, Q.; Wang, L.; Yan, X.; Kang, Y.; Wang, X.; Xie, S. A Method to Determine BeiDou GEO/IGSO Orbital Maneuver Time Periods. Sensors 2019, 19, 2675. [CrossRef]

22. Qin, Z.; Huang, G.; Zhang, Q.; Wang, L.; Yan, X.; Xie, S.; Cao, Y.; Wang, X. Precise Orbit Determination for BeiDou GEO/IGSO Satellites during Orbit Maneuvering with Pseudo-Stochastic Pulses. Remote Sens. 2019, 11, 2587. [CrossRef]

23. Hu, W. Fundamental Spacecraft Dynamics and Control; Wiley: Hoboken, NJ, USA, 2015. [CrossRef]

24. Fan, L. Research on Signal-In-Space Error Anomaly Detection and Performance Assessment of BDS Satellites; Chang'an University: Xi'an, China, 2018. 